revised CERN-TH.7400/94

hep-ph/9408395

\title{
ESTIMATES OF THE HIGHER-ORDER QCD CORRECTIONS: THEORY AND APPLICATIONS
}

\author{
Andrei L. Kataev 円 \\ Theoretical Physics Division, CERN, \\ CH-1211 Geneva 23, Switzerland \\ and \\ Valery V. Starshenko \\ Chair of General Physics, \\ Zaporozhye Industrial Institute \\ 330600 Zaporozhye, Ukraine
}

\begin{abstract}
We consider the further development of the formalism of the estimates of higher-order perturbative corrections in the Euclidean region, which is based on the application of the schemeinvariant methods, namely the principle of minimal sensitivity and the effective charges approach. We present the estimates of the order $O\left(\alpha_{s}^{4}\right)$ QCD corrections to the Euclidean quantities: the $e^{+} e^{-}$-annihilation $D$-function and the deep inelastic scattering sum rules, namely the non-polarized and polarized Bjorken sum rules and to the Gross-Llewellyn Smith sum rule. The results for the $D$-function are further applied to estimate the $O\left(\alpha_{s}^{4}\right)$ QCD corrections to the Minkowskian quantities $R(s)=\sigma_{\text {tot }}\left(e^{+} e^{-} \rightarrow\right.$ hadrons $) / \sigma\left(e^{+} e^{-} \rightarrow \mu^{+} \mu^{-}\right)$and $R_{\tau}=\Gamma\left(\tau \rightarrow \nu_{\tau}+\right.$ hadrons $) / \Gamma\left(\tau \rightarrow \nu_{\tau} \bar{\nu}_{e} e\right)$. The problem of the fixation of the uncertainties due to the $O\left(\alpha_{s}^{5}\right)$ corrections to the considered quantities is also discussed.
\end{abstract}

Shortened vesrion to submitted to the Proceedings of the QCD-94 Workshop, Montpellier, France, 7-13 July 1994, to be published in Nucl. Phys. B Proc. Suppl., ed. S. Narison

revised CERN-TH.7400/94

August-September 1994.

\footnotetext{
${ }^{1}$ On leave of absence from Institute for Nuclear Research of the Russian Academy of Sciences, 117312 Moscow, Russia
} 
I. The main aim of this work, which is based on the considerations of Refs. [1, 2], is to obtain the estimates of the higher-order QCD corrections to $R(s), R_{\tau}$ and deep-inelastic scattering sum rules using the improvement formula [3] of re-expansion of the expressions for the physical quantities obtained within the principle of minimal sensitivity (PMS) [3] and the effective charges (ECH) approach [4], which is equivalent a posteriori to scheme-invariant perturbation theory [5].

Of course, this approach to the estimation of the values of the uncalculated higher-order terms can not be considered as an alternative to direct analytical or numerical calculations. However, we hope that this method can give an idea of the possible values of the higher-order terms. This hope is based on the fact that the application of this method for the estimate of the four-loop corrections to the expression for $(g-2)_{e}$ [6] gave results which turned out to be in surprisingly good agreement with the latest results of the direct numerical calculations of Ref. [7] 2. The second argument in favour of this procedure came from its successful application for the analysis of the Drell-Yan cross section at the $O\left(\alpha_{s}^{2}\right)$-level [9]. Moreover, as will be demonstrated in our work, the re-expansion formalism of Ref. [3] is also working quite well in QCD at the $N N L O$ for at least three independent quantities, namely for the $e^{+} e^{-}$-annihilation $D$-function and the non-polarized and polarized Bjorken sum rules. Therefore, we prefer to consider these five facts not as a surprising accidental coincidence, but as an argument in favour of more detailed studies of the intrinsic features of this approach and its various applications.

In this work we further develop the re-expansion formalism of Ref. [3], deriving new terms in the corresponding improvement formula. The previously-known terms are used to obtain the estimates of the next-to-next-to-next-to-leading order $\left(N^{3} L O\right)$ QCD corrections to the $D$ function, the non-polarized Bjorken sum rule and polarized Bjorken sum rule, which is closely related by the structure of the corresponding perturbative series to the Gross-Llewellyn Smith sum rule. We will use the results obtained for the $D$-function to estimate the effects of the $N^{3} L O$ corrections to the perturbative series for the Minkowskian quantities $R(s)$ and $R_{\tau}$ by adding the explicitly calculable terms, previously discussed in Refs. [10, 11], supplementing thus the related considerations of Refs. [3], [12] by the additional input information. The derived new term in the improvement formula is applied to touch on the problem of fixing the values of the $O\left(\alpha_{s}^{5}\right)$-corrections to the analysed quantities.

II. Consider first the order $O\left(a^{N}\right)$ approximation of a Euclidean physical quantity

$$
D_{N}=d_{0} a\left(1+\sum_{i=1}^{N-1} d_{i} a^{i}\right)
$$

with $a=\alpha_{s} / \pi$ being the solution of the corresponding renormalization group equation for the $\beta$-function which is defined as

$$
\mu^{2} \frac{\partial a}{\partial \mu^{2}}=\beta(a)=-\beta_{0} a^{2}\left(1+\sum_{i=1}^{N-1} c_{i} a^{i}\right) .
$$

\footnotetext{
${ }^{2} \mathrm{~A}$ detailed re-consideration of the analysis of Ref. [6] and its generalization to five-loop order will be presented in a more detailed work [8].
} 
In the process of the concrete calculations of the coefficients $d_{i}, i \geq 1$ and $c_{i}, i \geq 2$, the $\overline{M S}$ scheme is commonly used. However, this scheme is not the unique prescription for fixing the RS ambiguities (for the recent discussions see e.g., Ref. [12]).

The PMS [3] and ECH [4] prescriptions stand out from various methods of treating schemedependence ambiguities. Indeed, they are based on the conceptions of the scheme-invariant quantities, which are defined as the combinations of the scheme-dependent coefficients in Eqs. (1) and (2). Both these methods pretend to be the "optimal" prescriptions, in the sense that they might provide better convergence of the corresponding approximations in the nonasymptotic regime, and thus allow an estimation of the uncertainties of the perturbative series in the definite order of perturbation theory. Therefore, applying these "optimal" methods, one can try to estimate the effects of the order $O\left(a^{N+1}\right)$-corrections starting from the approximations $D_{N}^{o p t}\left(a_{\text {opt }}\right)$ calculated in a certain "optimal" approach [3], [6], [13].

Let us following the considerations of Ref. [3] and re-expand $D_{N}^{\text {opt }}\left(a_{\text {opt }}\right)$ in terms of the coupling constant $a$ of the particular scheme

$$
D_{N}^{o p t}\left(a_{o p t}\right)=D_{N}(a)+\delta D_{N}^{o p t} a^{N+1}
$$

where

$$
\delta D_{N}^{o p t}=\Omega_{N}\left(d_{i}, c_{i}\right)+\Omega_{N}\left(d_{i}^{o p t}, c_{i}^{o p t}\right)
$$

are the numbers which simulate the coefficients of the order $O\left(a^{N+1}\right)$-corrections to the physical quantity, calculated in the particular initial scheme, say the $\overline{M S}$-scheme.

The explicit form of the coefficients $\Omega_{i}$ can be obtained following the considerations of Ref. [3]. We present here the final already known expressions [3]:

$$
\begin{gathered}
\Omega_{2}=d_{0} d_{1}\left(c_{1}+d_{1}\right), \\
\Omega_{3}=d_{0} d_{1}\left(c_{2}-\frac{1}{2} c_{1} d_{1}-2 d_{1}^{2}+3 d_{2}\right) .
\end{gathered}
$$

and the new term $\Omega_{4}$ evaluated by us:

$$
\Omega_{4}=\frac{d_{0}}{3}\left(3 c_{3} d_{1}+c_{2} d_{2}-4 c_{2} d_{1}^{2}+2 c_{1} d_{1} d_{2}-c_{1} d_{3}+14 d_{1}^{4}-28 d_{1}^{2} d_{2}+5 d_{2}^{2}+12 d_{1} d_{3}\right)
$$

which reproduces the renormalization-group controllable logarithmic terms at the five-loop level [14].

It should be stressed that in the ECH approach $d_{i}^{E C H} \equiv 0$ for all $i \geq 2$. Therefore one gets the following expressions for the $N N L O$ and $N^{3} L O$ corrections in Eq. (3):

$$
\begin{gathered}
\delta D_{2}^{E C H}=\Omega_{2}\left(d_{1}, c_{1}\right) \\
\delta D_{3}^{E C H}=\Omega_{3}\left(d_{1}, d_{2}, c_{1}, c_{2}\right) \\
\delta D_{4}^{E C H}=\Omega_{4}\left(d_{1}, d_{2}, d_{3}, c_{1}, c_{2}, c_{3}\right)
\end{gathered}
$$


In order to find similar corrections to Eq.(3) in the N-th order of perturbation theory starting from the PMS approach [3], it is necessary to use the relations obtained in Ref. [15] between the coefficients $r_{i}^{P M S}$ and $c_{i}^{P M S}(i \geq 1)$ in the expression for the order $O\left(a_{P M S}^{N}\right)$ approximation $D_{N}^{P M S}\left(a_{P M S}\right)$ of the physical quantity under consideration. The corresponding corrections have the following form:

$$
\begin{gathered}
\delta D_{2}^{P M S}=\delta D_{2}^{E C H}+\frac{d_{0} c_{1}^{2}}{4} \\
\delta D_{3}^{P M S}=\delta D_{3}^{E C H} .
\end{gathered}
$$

Notice the identical coincidence of the $N^{3} L O$ corrections obtained starting from both the PMS and ECH approaches. A similar observation was made in Ref. [6] using different (but related) considerations.

In the fourth order of perturbation theory the additional contribution to $\delta D_{4}^{P M S}$ has more complicated structure. Indeed, the expression for $\Omega_{4}\left(d_{i}^{P M S}, c_{i}^{P M S}\right)$ in Eq. (4) reads:

$$
\Omega_{4}\left(d_{i}^{P M S}, c_{i}^{P M S}\right)=\frac{d_{0}}{3}\left[\frac{1}{4} c_{1} c_{3}^{P M S}-\frac{4}{81}\left(c_{2}^{P M S}\right)^{2}-\frac{5}{81} c_{1}^{2} c_{2}^{P M S}+\frac{7}{648} c_{1}^{4}\right]
$$

where

$$
c_{2}^{P M S}=\frac{9}{8}\left(d_{2}+c_{2}-d_{1}^{2}-c_{1} d_{1}+\frac{7}{36} c_{1}^{2}\right)+O(a)
$$

and

$$
c_{3}^{P M S}=4\left(d_{3}+\frac{1}{2} c_{3}-c_{2} d_{1}-3 d_{1} d_{2}+2 d_{1}^{3}\right)+\frac{1}{2} c_{1}\left(d_{2}+c_{2}+3 d_{1}^{2}-c_{1} d_{1}+\frac{1}{108} c_{1}^{2}\right)+O(a) .
$$

The expressions for Eqs. (14)- (16) are pure numbers, which do not depend on the choice of the initial scheme. Note, that we have checked that in the case of the consideration of the perturbative series for $(g-2)_{e}$ and $(g-2)_{\mu}$ the numerical values of $\Omega_{4}\left(d_{i}^{P M S}, c_{i}^{P M S}\right)$ are small and thus the a posteriori approximate equivalence of the ECH and PMS approaches is preserved for the quantities under consideration at this level also [8]. We think that this feature is also true in QCD.

III. Consider now the familiar characteristic of the $e^{+} e^{-} \rightarrow \gamma \rightarrow$ hadrons process, namely the $D$-function defined in the Euclidean region:

$$
D\left(Q^{2}\right)=Q^{2} \int_{0}^{\infty} \frac{R(s)}{\left(s+Q^{2}\right)^{2}} d s
$$

Its perturbative expansion has the following form:

$$
D\left(Q^{2}\right)=3 \Sigma Q_{f}^{2}\left[1+a+\sum_{i \geq 1} d_{i} a^{i+1}\right]+\left(\Sigma Q_{f}\right)^{2}\left[\tilde{d}_{2} a^{3}+O\left(a^{4}\right)\right]
$$

where $Q_{f}$ are the quark charges, and the structure proportional to $\left(\Sigma Q_{f}\right)^{2}$ comes from the light-by-light-type diagrams. The coefficients $d_{1}$ and $d_{2}, \tilde{d}_{2}$ were calculated in the $\overline{M S}$-scheme in Refs. [16] and [17, 18] respectively. Following the proposals of Ref. [19], we will treat the light-by-light-type term in Eq. (18) separately from the "main" structure of the $D$-function, 
which is proportional to the quark-parton expression $D^{Q P}\left(Q^{2}\right)=3 \Sigma Q_{f}^{2}$. In fact, one can hardly expect that it is possible to predict higher-order coefficients $\tilde{d}_{i}, i \geq 3$ of the second structure in Eq. (17) using the only explicitly-known term $\tilde{d}_{2}$. Therefore we will neglect the light-by-lighttype structure as a whole in all our further considerations. This approximation is supported by the relatively tiny contribution of the second structure of Eq. (17) to the final NNLO correction to the $D$-function.

The next important ingredient of our analysis is the QCD $\beta$-function (2), which is known in the MS-like schemes at the NNLO level [20].

Using now the perturbative expression for the $D$-function, one can obtain the perturbative expression for $R(s)$, namely

$$
R(s)=3 \Sigma Q_{f}^{2}\left[1+a_{s}+\sum_{i \geq 1} r_{i} a_{s}^{i+1}\right]+\left(\Sigma Q_{f}\right)^{2}\left[\tilde{r}_{2} a_{s}^{3}+\ldots\right]
$$

where $a_{s}=\bar{\alpha}_{s} / \pi, r_{1}=d_{1}, r_{2}=d_{2}-\pi^{2} \beta_{0}^{2} / 3, \tilde{r}_{2}=\tilde{d}_{2}$ and $r_{3}=d_{3}-\pi^{2} \beta_{0}^{2}\left(d_{1}+\frac{5}{6} c_{1}\right)$. The corresponding $\pi^{2}$-terms come from the analytic continuation of the Euclidean result for the $D$-function to the physical region. The effects of the higher-order $\pi^{2}$-terms were discussed in detail in Ref. [10]. For example, the corresponding expression for the $r_{4}$-term reads :

$$
r_{4}=d_{4}-\pi^{2} \beta_{0}^{2}\left(2 d_{2}+\frac{7}{3} c_{1} d_{1}+\frac{1}{2} c_{1}^{2}+c_{2}\right)+\frac{\pi^{4}}{5} \beta_{0}^{4}
$$

The perturbative expression for $R_{\tau}$ is defined as

$$
R_{\tau}=2 \int_{0}^{M_{\tau}^{2}} \frac{d s}{M_{\tau}^{2}}\left(1-s / M_{\tau}^{2}\right)^{2}\left(1+2 s / M_{\tau}^{2}\right) \tilde{R}(s) \simeq 3\left[1+a_{\tau}+\sum_{i \geq 1} r_{i}^{\tau} a_{\tau}^{i+1}\right]
$$

where $a_{\tau}=\alpha_{s}\left(M_{\tau}^{2}\right) / \pi$ and $\tilde{R}(s)$ is $R(s)$ with with $f=3,\left(\Sigma Q_{f}\right)^{2}=0,3 \Sigma Q_{f}^{2}$ substituted for $3 \Sigma\left|V_{f f^{\prime}}\right|^{2}$ and $\left|V_{u d}\right|^{2}+\left|V_{u s}\right|^{2} \approx 1$.

It was shown in Ref. [11] that it is convenient to express the coefficients of the series (24) through those ones of the series (18) for the $D$-function in the following form :

$$
\begin{aligned}
r_{1}^{\tau} & =d_{1}^{\overline{M S}}(f=3)+g_{1} \\
r_{2}^{\tau} & =d_{2}^{\overline{M S}}(f=3)+g_{2} \\
r_{3}^{\tau} & =d_{3}^{\overline{M S}}(f=3)+g_{3}
\end{aligned}
$$

where in our notations

$$
\begin{aligned}
& g_{1}=-\beta_{0} I_{1}=3.563 \\
& g_{2}=-\left[2 d_{1}+c_{1}\right] \beta_{0} I_{1}+\beta_{0}^{2} I_{2}=19.99 \\
& g_{3}=-\left[3 d_{2}+2 d_{1} c_{1}+c_{2}\right] \beta_{0} I_{1}+\left[3 d_{1}+\frac{5}{2} c_{1}\right] \beta_{0}^{2} I_{2}-\beta_{0}^{3} I_{3}=78.00
\end{aligned}
$$


and $I_{k}$ are defined and calculated in Ref. [11]. Their analytical expressions read: $I_{1}=-19 / 12$, $I_{2}=265 / 72-\pi^{2} / 3$ and $I_{3}=-3355 / 288+19 \pi^{2} / 12$. One of the pleasant features of Eqs.(22) is that they are absorbing all effects of the analytical continuation.

Following the lines of Ref. 11] we derive the corresponding expression for the coefficient $r_{4}^{\tau}$ :

$$
r_{4}^{\tau}=d_{4}^{\overline{M S}}(f=3)+g_{4}
$$

where

$$
\begin{aligned}
g_{4}= & -\left[4 d_{3}+3 d_{2} c_{1}+2 d_{1} c_{2}+c_{3}\right] \beta_{0} I_{1}+\left[6 d_{2}+7 c_{1} d_{1}+\frac{3}{2} c_{1}^{2}+3 c_{2}\right] \beta_{0}^{2} I_{2} \\
& -\left[4 d_{1}+\frac{13}{3} c_{1}\right] \beta_{0}^{3} I_{3}+\beta_{0}^{4} I_{4}=3.562 c_{3}(f=3)+14.247 d_{3}(f=3)-466.818
\end{aligned}
$$

and $I_{4}=41041 / 864-265 \pi^{2} / 36+\pi^{4} / 5 \approx-5.668$.

In order to estimate the values of the order $O\left(a^{3}\right), O\left(a^{4}\right)$ and $O\left(a^{5}\right)$ corrections to $R(s)$ and $R_{\tau}$, we will apply Eqs. (5) - (10) in the Euclidean region to the perturbative series for the $D$-function and then obtain the estimates we are interested in using Eqs. (18), (19), (21)-(24). This is the new ingredient of this analysis, which distinguishes it from the related ones of Refs. [3], [12].

We now recall the perturbative expression for the non-polarized Bjorken deep-inelastic scattering sum rule

$$
\operatorname{BjnSR}=\int_{0}^{1} F_{1}^{\bar{v} p-v p}\left(x, Q^{2}\right) d x=1-\frac{2}{3} a\left(1+\sum_{i \geq 1} d_{i} a^{i}\right)
$$

where the coefficients $d_{1}$ and $d_{2}$ are known in the $\overline{M S}$ scheme from the results of calculations of Ref. [21] and Ref. [22]. The expression for the polarized Bjorken sum rule BjpSR has the following form:

$$
\operatorname{BjpSR}=\int_{0}^{1} g_{1}^{e p-e n}\left(x, Q^{2}\right) d x=\frac{1}{3}\left|\frac{g_{A}}{g_{V}}\right|\left[1-a\left(1+\sum_{i \geq 1} d_{i} a^{i}\right)\right]
$$

where the coefficients $d_{1}$ and $d_{2}$ were explicitly calculated in the $\overline{M S}$ scheme in Refs. [23] and [24] respectively.

It is also worth emphasizing that, in spite of the identical coincidence of the $N L O$ correction to the Gross-Llewellyn Smith sum rule $G L S S R=(1 / 2) \int_{0}^{1} F_{3}^{\bar{\nu} p+\nu p}\left(x, Q^{2}\right) d x$ with the ones for the $\operatorname{BjpSR}$ [23] the corresponding NNLO correction differs from the one for the $\operatorname{Bjp} S R$ by the contributions of the light-by-light-type terms typical of the GLSSR [24]. Since these lightby-light-type terms appear for the first time at the $N N L O$, it is impossible to predict the value of the light-by-light-type contribution at the $N^{3} L O$ level using the corresponding $N N L O$ terms as the input information. However, noticing that at the $N N L O$ level the corresponding light-by-light-type contributions are small [24], we will assume that the similar contributions are small at the $N^{3} L O$ level also. Only after this assumption can our estimates of the $N N L O$ 
and $N^{3} L O$ corrections to the BjpSR be considered also as the estimates of the corresponding corrections in the perturbative series for the GLSSR .

IV. The estimates of the coefficients of the order $O\left(a^{3}\right), O\left(a^{4}\right)$ and $O\left(a^{5}\right)$ QCD corrections to the $D$-function, $R(s)$, BjnSR and BjpSR/GLSSR obtained from the ECH-improved expressions are presented in Tables $1-4$ respectively. Due to the complicated $f$-dependence of the coefficients $\Omega_{2}, \Omega_{3}, \Omega_{4}$ in Eqs. (5)-(7), we are unable to predict the explicit $f$-dependence of the corresponding coefficients in the form respected by perturbation theory. The results are presented for the fixed number of quark flavours $1 \leq f \leq 6$ and are normalized to the $\overline{M S}$-scheme.

\begin{tabular}{|c|c|c|c|c|}
\hline$f$ & $d_{2}^{\text {ex }}$ & $d_{2}^{\text {est }}$ & $d_{3}^{\text {est }}$ & $d_{4}^{\text {est }}-c_{3} d_{1}$ \\
\hline 1 & 14.11 & 7.54 & 75 & 474 \\
\hline 2 & 10.16 & 6.57 & 50 & 261 \\
\hline 3 & 6.37 & 5.61 & 27.5 & 111 \\
\hline 4 & 2.76 & 4.68 & 8 & 23 \\
\hline 5 & -0.69 & 3.77 & -8 & -15 \\
\hline 6 & -3.96 & 2.88 & -21 & -1.8 \\
\hline
\end{tabular}

Table 1 : The results of estimates of the $N N L O, N^{3} L O$ and $N^{4} L O$ corrections in the series for the $D$-functions.

\begin{tabular}{|c|c|c|c|c|}
\hline$f$ & $r_{2}^{\text {ex }}$ & $r_{2}^{\text {est }}$ & $r_{3}^{\text {est }}$ & $r_{4}^{\text {est }}-c_{3} d_{1}$ \\
\hline 1 & -7.84 & -14.41 & -166 & -1748 \\
\hline 2 & -9.04 & -12.63 & -147 & -1156 \\
\hline 3 & -10.27 & -11.03 & -128 & -669 \\
\hline 4 & -11.52 & -9.58 & -112 & -263 \\
\hline 5 & -12.76 & -8.29 & -97 & 64 \\
\hline 6 & -14.01 & -7.17 & -83 & 334 \\
\hline
\end{tabular}

Table 2: The results of estimates of the $N N L O, N^{3} L O$ and $N^{4} L O$ corrections in the series for $R(s)$. 


\begin{tabular}{|c|c|c|c|c|}
\hline$f$ & $d_{2}^{\text {ex }}$ & $d_{2}^{\text {est }}$ & $d_{3}^{\text {est }}$ & $d_{4}^{\text {est }}-c_{3} d_{1}$ \\
\hline 1 & 44.97 & 39.62 & 424 & 4177 \\
\hline 2 & 36.19 & 33.28 & 303 & 2619 \\
\hline 3 & 27.89 & 27.37 & 200 & 1481 \\
\hline 4 & 20.07 & 21.91 & 114 & 671 \\
\hline 5 & 12.72 & 16.91 & 44 & 137 \\
\hline 6 & 5.85 & 12.39 & -10 & -148 \\
\hline
\end{tabular}

Table 3: The results of estimates of the $N N L O, N^{3} L O$ and $N^{4} L O$ corrections in the series for BjnSR.

\begin{tabular}{|c|c|c|c|c|}
\hline$f$ & $d_{2}^{\text {ex }}$ & $d_{2}^{\text {est }}$ & $d_{3}^{\text {est }}$ & $d_{4}^{\text {est }}-c_{3} d_{1}$ \\
\hline 1 & 34.01 & 27.25 & 290 & 2557 \\
\hline 2 & 26.93 & 23.11 & 203 & 1572 \\
\hline 3 & 20.21 & 19.22 & 130 & 854 \\
\hline 4 & 13.84 & 15.57 & 68 & 342 \\
\hline 5 & 7.83 & 12.19 & 18 & 27 \\
\hline 6 & 2.17 & 9.08 & -22 & -135 \\
\hline
\end{tabular}

Table 4: The results of estimates of the $N N L O, N^{3} L O$ and $N^{4} L O$ corrections in the series for BjpSR and GLSSR.

The presented $N N L O$ estimates are in reasonable agreement with the results of the explicit calculations. The best agreement is achieved for $f=3$ numbers of flavours. It can be shown that the difference with the results obtained staring from the PMS improved expression is rather small [1, 2].

In order to address the problem of fixing the values of the $O\left(a^{5}\right)$ QCD corrections to the considered Euclidean quantities, Eqs. (7), (10) with explicitly calculated NLO and NNLO coefficients $d_{1}, d_{2}, c_{1}, c_{2}$ were applied. The values of the coefficients $d_{3}$ were fixed by using the estimates of the $N^{3} L O$ coefficients the determined from Eqs. (6), (9). To estimate the nextto-next-to-next-to-next-to-leading order $\left(N^{4} L O\right)$ coefficient of $R(s)$, which is related to the $N^{4} \mathrm{LO}$ coefficient $d_{4}$ of the $D$-function, the explicitly calculated terms in Eq.(19) were taken into account. However, the expressions for $\Omega_{4}$ in Eq. (7) depend also on the four-loop coefficient $c_{3}$ of the QCD $\beta$-function which is unknown at present. Therefore, in Tables 1-4 we presented the estimates of the combinations $d_{4}-d_{1} c_{3}$ and $r_{4}-r_{1} c_{3}$. Another existing uncertainty in the value of $N^{3} L O$ coefficients $d_{3}$ is fixed by the assumption that the real values of these coefficients do not significantly differ from the $N^{3} L O$ estimates obtained by us. However, in the case of the $D$-function with $f=3$ numbers of flavours we present the more detailed expression of $d_{4}$ :

$$
d_{4}^{e s t}(f=3)=1.64 c_{3}(f=3)+5.97 d_{3}(f=3)-52.66 .
$$

In order to obtain the corresponding estimate from Table 1, Eq.(27) should be supplemented by the $N^{3} L O$-estimate $d_{3}^{\text {est }}(f=3)=27.5$ first obtained in Ref. [1]. This result is in good agreement 
with the "geometric progression" assumption $d_{3}(f=3) \approx d_{2}^{2}(f=3) / d_{1}(f=3) \approx 25$ of Ref. [11]. Our result was recently supported [25] by the phenomenological analysis of the ALEPH data for $R_{\tau}$.

Amongst other most interesting results of our analysis are the estimates of the $N^{3} L O$ and $N^{4} L O$ corrections to $R(s)$ for $f=5$ numbers of flavours. Taking $\alpha_{s}\left(M_{Z}\right) \approx 0.12$, we get the estimate of the corresponding $N^{3} L O$ contribution to $\Gamma\left(Z^{0} \rightarrow\right.$ hadrons $)$

$$
\left(\delta \Gamma_{Z^{0}}\right)_{N^{3} L O} \approx-97\left(a\left(M_{Z}\right)\right)^{4} \approx-2 \times 10^{-4} .
$$

The comparison of the results of Table 1 with those of Table 2 demonstrate that the $\pi^{2}$-effects give dominating contributions to the coefficients of $R(s)$ and $\Gamma_{Z^{0}}$. Indeed, at the $N^{4} L O$-level they are also larger than the estimated coefficient $d_{4}$ of the Euclidean $D$-function. Assuming that $c_{3}(f=5)=c_{2}(f=5)^{2} / c_{1}(f=5) \approx 1.715$ we arrive at the following less substantiated estimate of the corresponding $N^{4} L O$ contributions, namely

$$
\left(\delta \Gamma_{Z^{0}}\right)_{N^{4} L O} \approx 64\left(a\left(M_{Z}\right)\right)^{5} \approx 5 \times 10^{-6}
$$

The way of fixation of the value of $c_{3}$ used above is not applicable for the case of $f=6$, since we expect that in this case its real value is negative.

Using now the relations derived in Refs. [26, 11] between the coefficients of the $D$-function and $R_{\tau}$ and taking $\alpha_{s}\left(M_{\tau}\right) \approx 0.36$ [26] we get the following estimate of the $N^{3} L O$ contribution to $R_{\tau}$ :

$$
\left(\delta R_{\tau}\right)_{N^{3} L O} \approx 105.5 a_{\tau}^{4} \approx 1.8 \times 10^{-2} .
$$

This estimate is more precise than those presented in Refs. [26] and [11], namely $\delta R_{\tau}= \pm 130 a_{\tau}^{4}$ [26] and $\delta R_{\tau}=(78 \pm 25) a_{\tau}^{4}$ [11], and is smaller than the result of applying the Padé resummation technique directly to $R_{\tau}$, namely $\delta R_{\tau}=133 a_{\tau}^{4}$ [27].

Generalizing the considerations of Ref. 11] to the order $O\left(a_{\tau}^{5}\right)$-level and taking $c_{3}(f=3) \approx$ $c_{2}(f=3)^{2} / c_{1}(f=3) \approx 11$ we get the estimate of the $N^{4} L O$ corrections to $R_{\tau}$ [2]. This estimate reads [2]:

$$
\left(\delta R_{\tau}\right)_{N^{4} L O} \approx 94 a_{\tau}^{5} \approx 1.8 \times 10^{-3}
$$

V. Let us emphasize that the results for $R(s)$ and $R_{\tau}$ were obtained by us from those of the $D$-function after taking into account explicitly calculable terms of the analytic continuation to the Minkowskian region, discussed in detail in Ref. [28]. In principle, one can try to study the application of the procedure used by us directly to $R(s)$ and $R_{\tau}$ in the Minkowskian region. We checked that in the case of applications of Eqs. (5)-(7) with the substitution of $d_{i}=r_{i}$ or $d_{i}=r_{i}^{\tau}$ one can reproduce the same values of the Eucledian coefficients $d_{i}$ but the structure of the high order $\pi^{2}$-terms will not agree with the explicitly known results. A similar problem will also definitely arise in the case of more rigorous studies of the applicability of the Padé resummation methods for the Minkowskian quantities $R(s)$ and $R_{\tau}$ (for the studies existing at present see Refs. [27]). We think that these analyses should be supplemented by the construction of the Padé approximants directly to the $D$-function. In our case it is possible to reproduce our results $R(s)$ and $R_{\tau}$ by means of application of a similar technique directly in the Minkowskian region 
after modifications of Eqs. (5)-(7) in the Minkowskian region by adding concrete calculable $\pi^{2}$-dependent and scheme-independent factors.

\section{Acknowledgements}

We are grateful to R.N. Faustov for attracting our attention to the necessity for detailed consideration of the results of Ref. [6] at the preliminary stage of our similar QED studies, which will be presented elsewhere, and to J. Chýla, F. Le Diberder and G. Marchesini for discussions and to J. Ellis for the useful comments on the earlier version of this paper.

\section{References}

[1] A.L. Kataev and V.V. Starshenko, preprint CERN-TH.7198/94; Proc. of the Workshop "QCD at LEP: Determination of $\alpha_{s}$ from Inclusive Observables", Aachen, Germany, 11 April 1994, eds. W. Bernreuther and S. Bethke, Aachen Report PITHA 94/33 (1994)

[2] A.L. Kataev and V.V. Starshenko, submitted for publication.

[3] P.M. Stevenson, Phys.Rev. D23 (1981) 2916.

[4] G. Grunberg, Phys. Lett. B221 (1980) 70; Phys.Rev. D29 (1984) 2315.

[5] A. Dhar and V. Gupta, Phys.Rev. D29 (1984) 2822;

V. Gupta, D.V. Shirkov and O.V. Tarasov, Int. J. Mod. Phys.A6 (1991) 3381.

[6] J. Kubo and S. Sakakibara, Z. Phys. C14 (1982) 345.

[7] T. Kinoshita and W.B. Lindquist, Phys.Rev. D42 (1990) 636.

[8] A.L. Kataev and V.V. Starshenko, to be published.

[9] P. Aurenche, R. Bair and M. Fontannaz, Z. Phys. C48 (1990) 143.

[10] J.D. Bjorken, report SLAC-PUB-5103 (1989).

[11] F. Le Diberder and A. Pich, Phys. Lett. B286 (1992) 147.

[12] J.H. Field, Ann. Phys. 226 (1993) 209.

[13] V.V. Starshenko and R.N. Faustov, JINR Rapid Communications 7 (1985) 39.

[14] A.L. Kataev, Phys. Lett. B284 (1992) 401.

[15] M.R.Pennington, Phys.Rev. D26 (1986) 2048.

[16] K.G. Chetyrkin, A.L. Kataev and F.V. Tkachov, Phys. Lett. 85B (1979) 277;

M. Dine and J. Sapirstein, Phys. Rev. Lett. 43 (1979) 668;

W. Celmaster and R. Gonsalves, Phys. Rev. Lett. 44 (1980) 560. 
[17] S.G. Gorishny, A.L. Kataev and S.A. Larin, Phys. Lett. B259 (1991) 144; Pisma Zhetf 53 (1991) 121.

[18] L.R. Surguladze and M.A. Samuel, Phys. Rev. Lett. 66 (1991) 560; 66 (1991) 2416 (Erratum).

[19] S. Brodsky, G.P. Lepage and P.B. Mackenzie, Phys.Rev. D28 (1983) 228;

Hung Jung Lu, Phys.Rev. D45 (1992) 1217.

[20] O.V. Tarasov, A.A. Vladimirov and A.Yu. Zharkov, Phys. Lett. 93B (1980) 429;

S.A. Larin and J.A.M. Vermaseren, Phys. Lett. B303 (1993) 334.

[21] K.G. Chetyrkin, S.G. Gorishny, S.A. Larin and F.V. Tkachov, Phys. Lett. 137B (1984) 230.

[22] S.A. Larin, F.V. Tkachov and J.A.M. Vermaseren, Phys. Rev. Lett. 66 (1991) 862.

[23] S.G. Gorishny and S.A. Larin, Phys. Lett. B172 (1986) 109;

E.B. Zijlstra and W. van Neerven, Phys. Lett. B297 (1992) 377.

[24] S.A. Larin and J.A.M. Vermaseren, Phys. Lett. B259 (1991) 345.

[25] F. Le Diberder, talk at the QCD-94 Workshop, Montpellier, France, July 1994, to appear in the Proceedings, ed. S. Narison.

[26] E. Braaten, S. Narison and A. Pich, Nucl. Phys. B373 (1992) 581.

[27] M.A.Samuel and G.Li, preprint SLAC-PUB-6379 (1993); Phys. Lett. B331 (1994) 114.

[28] A.A. Pivovarov, Nuov. Cim. A105 (1992) 813;

A.A. Pivovarov Z. Phys. C53 (1992) 461. 